Review

\title{
Immunotherapies: The Blockade of Inhibitory Signals
}

\author{
Yan-Ling $\mathrm{Wu}^{1}{ }^{\bowtie}$, Jing Liang${ }^{2}$, Wen Zhang ${ }^{2}$, Yoshimasa Tanaka ${ }^{3}$, and Hiroshi Sugiyama ${ }^{4}$ \\ 1. Virus Inspection Department of Zhejiang Provincial Center for Disease Control and Prevention, 630 Xincheng Road, Hangzhou, 310051 , \\ China. \\ 2. Department of Biopharmaceutical Sciences, College of Pharmaceutical Sciences, Zhejiang University of Technology, 18 Chaowang Road, \\ Hangzhou, 310014, China. \\ 3. Center for Innovation in Immunoregulative Technology and Therapeutics, Graduate School of Medicine, Kyoto University, Kyoto, \\ 606-8501, Japan. \\ 4. Department of Chemistry, Graduate School of Science, Kyoto University, Sakyo-ku, Kyoto 606-8502, Japan.
}

\begin{abstract}
$\triangle$ Corresponding author: Yan-Ling Wu, Virus Inspection Department of Zhejiang Provincial Center for Disease Control and Prevention, 630 Xincheng Road, Hangzhou, 310051, PR China; Tel: +86-571-87115282; Fax: +86-571-87115282; e-mail: ylwu@cdc.zj.cn; Wen Zhang, Department of Biopharmaceutical Sciences, College of Pharmaceutical Sciences, Zhejiang University of Technology, 18 Chaowang Road, Hangzhou, 310014, PR China; e-mail: wzhang63@zjut.edu.cn.

() Ivyspring International Publisher. This is an open-access article distributed under the terms of the Creative Commons License (http://creativecommons.org/ licenses/by-nc-nd/3.0/). Reproduction is permitted for personal, noncommercial use, provided that the article is in whole, unmodified, and properly cited.
\end{abstract}

Received: 2012.09.22; Accepted: 2012.11.06; Published: 2012.11.17

\begin{abstract}
T lymphocytes require signaling by the $T$ cell receptor and by nonclonotypic cosignaling receptors. The costimulatory and inhibitory signals profoundly influence the course of immune responses by amplifying or reducing the transcriptional effects of $T$ cell receptor triggering. The inhibitory receptors such as CTLA-4, PD-I, and BTLA have recently drawn much attention as potential targets for immunotherapies. This review focuses on the progress that has been made with the mentioned receptors in the field of immunotherapies for autoimmune diseases, malignancies, infectious diseases, and transplantation.
\end{abstract}

Key words: Inhibitory receptors, CTLA-4, PD-1, BTLA, immunotherapy.

\section{Introduction}

$\mathrm{T}$ cell recognition of antigenic peptide/major histocompatibility complex plays a pivotal role in the initiation and regulation of adaptive immunity. $\mathrm{T}$ cell receptor (TCR) signaling pathway alone is, however, insufficient for antigen-specific $\mathrm{T}$ cell responses [1], and a second pathway is required for defining and regulating the fine specificity of the responses. Costimulatory signals lead to effective cell activation, cytokine production, proliferation, and survival. Interruption of the costimulatory pathway not only results in the suppression of the immune responses, but in some cases induces antigen-specific unresponsiveness or tolerance.

A primary costimulatory signal is delivered through CD28 glycoprotein, which is capable of forming homodimers, allowing for interactions with either CD80 (B7-1) or CD86 (B7-2) expressed on antigen-presenting cells (APCs). Inducible costimulator
(ICOS, CD278, also known as H4) is a costimulatory receptor homologous to $\mathrm{CD} 28$ family and engages its ligand, ICOSL (CD275, B7-H2), expressed on the surface of lymphoid cells as well as nonlymphoid cells. In an attempt to develop novel strategies for enhancing immune responses in immunocompromized hosts with malignancies, agonistic monoclonal antibodies (mAbs) specific to costimulatory receptors appeared to be promising in animal models. Catastrophic systemic organ failure, however, occurred in the phase I clinical trial of an agonistic anti-CD28 mAb, which has hampered the development of immunomodulatory mAbs acting on costimulatory receptors.

In contrast to CD28 family receptors, cytotoxic T-lymphocyte antigen 4 (CTLA-4, CD152), programmed death-1 (PD-1, CD279), and B and T cell attenuator (BTLA, CD272) are inhibitory receptors which deliver negative signals in $\mathrm{T}$ cells. The inhibi- 
tory signals attenuate TCR-mediated signals, leading to decreased cell proliferation, cytokine production, and cell cycle progression. Hence, recently much attention has been focused on the blockade of the inhibitory signals as a means of immunopotentiation in immune diseases, infectious diseases, and malignancies.

\section{Structure and function of CTLA-4}

CTLA-4 is transcriptionally induced following T cell activation and shares about $30 \%$ identity with CD28 at the amino acids level [2-9]. This inhibitory receptor molecule is a type I transmembrane protein with a single V-set (variable domain-like-set) immunoglobulin superfamily (IgSF) extracellular domain, a transmembrane region and a cytoplasmic region. Whereas the cytoplasmic tail of CTLA-4 contains a YVKM motif, a typical immunoreceptor tyrosine-based inhibitory motif (ITIM), the molecules and the mechanism involved in this negative signal pathway has not been fully elucidated [8].

As opposed to CD86 monomer engagement with CD28 homodimer, the CTLA-4 homodimer preferentially engages with CD80 homodimer, with the affinity of CD86 for CTLA-4 being only $8 \%$ of that of CD80. A canonical motif, MYPPPY, is present in the extracellular domain of CTLA-4 and this sequence is involved in the binding between CTLA- 4 and CD80 on the basis of X-ray crystallography. The CTLA-4/CD80 binding interface is formed by orthogonal packing between the front sheets from each molecule and buries a total surface area of $1,200-1,300 \AA^{2}$. Since CD80 forms a homodimer as a consequence of packing between the back $\beta$-sheets, two CTLA- 4 molecules interact with the respective front $\beta$-sheets distal to the CD80 homodimer interface, leading to a divalent nature of CD80 molecule and consequently, a lattice-like feature of CTLA-4/CD80 complex. This distinct quaternary structure further implies that CTLA-4/CD80 engagement might provide adhesive interactions required for a biologically optimal response at the immunological synapse.

In CTLA-4 deficient mice, a severe autoimmune phenotype develops with death occurring 3-4 weeks after birth as a result of multiorgan destruction, demonstrating that CTLA-4 plays a pivotal role in the regulation of autoreactive and potentially detrimental peripheral $\mathrm{T}$ cell responses [4]. CTLA-4 negatively modulates IL-2 production, IL-2 receptor expression, and cell cycle progression of activated $\mathrm{T}$ cells. The inhibitory signal delivered by CTLA- 4 can be modulated by a number of factors, such as a concomitant signal via CD28 [5], the strength of the TCR signal, and the activation state of APC. In addition to the inhibitory functions in activated $\mathrm{CD}^{+} \mathrm{T}$ cells and $\mathrm{CD}^{+} \mathrm{T}$ cells, CTLA- 4 expressed in Foxp $3^{+}$regulatory $\mathrm{T}$ cells (Tregs) plays important roles in maintaining immunological self-tolerance and immune homeostasis. Tregs constitutively expressing CTLA-4 regulate effector T cells in trans, as exhausting Tregs in mice can provoke various autoimmune diseases [10]. It was recently demonstrated that Treg-specific CTLA- 4 deficiency impaired in vivo and in vitro suppressive function of Tregs, which appeared to be mediated by down-regulation of CD80 and CD86 expression on dendritic cells (DCs) [11], although the mechanism by which CTLA-4 enhanced the immunosuppressive function of Tregs was not fully clarified.

\section{Therapeutic application of CTLA-4}

The affinity of CD80/CD86 for CTLA-4 is higher than that for CD28, an antagonistic CTLA-4-Ig fusion protein, which is composed of the extracellular domain of CTLA- 4 and the FC region of the immunoglobulin IgG1 (Ig), can be used to efficiently inhibit CD28/CD80/CD86 signaling pathway and prevent the full activation of $\mathrm{T}$ cells. It was shown that CTLA-4-Ig ameliorated both the clinical and histopathologic manifestations of a joint disease in a collagen induced arthritis model [12]. In RA patients, abatacept (a clinical-grade CTLA-4-Ig preparation) improves the signs and symptoms of RA and inhibits the progression of joint damages [13]. The fusion protein is reported to enhance the function of Tregs in RA patients [14], which may also contribute to the suppression of $\mathrm{T}$ cell hyperactivation. CTLA-4-Ig can thus be utilized for the treatment of various autoimmune diseases, such as systemic lupus [11], experimental allergic encephalitis (EAE) [15] and collagen induced arthritis [12].

The attenuation of $\mathrm{T}$ cell hyperactivation during graft-versus-host diseases (GVHD) after transplantation by CTLA-4-Ig can be also considered as a therapeutic strategy, because several anti-CD28 antagonistic mAbs have been successfully utilized in animal models of renal allograft, heart allograft, and liver allograft [16-22]. It is of note that monotherapy with CTLA-4-Ig can lead to cardiac graft acceptance for more than 100 days in a mouse model [23]. Furthermore, porcine CTLA-4-Ig prolongs islet exnografts in rats with an increased serum level of IFN- $\gamma$ and a decreased serum level of IL-4 [24] (Table 1).

On the contrary, blocking monoclonal antibodies (mAbs) specific to CTLA-4 inhibits the CTLA-4 signaling pathway by binding to CD80/CD86 expressed on APCs and subsequently enhances $\mathrm{T}$ cell activation. The antagonistic mAbs are thus expected to be used for the enhancement of $\mathrm{T}$ cell cytotoxicity against tu- 
mor cells (Table 2). The anti-tumor effect of an anti-CTLA-4 mAb was first reported in murine models of colon carcinoma and fibrosarcoma in 1996 [25]. The mAb-mediated blockade of CTLA-4 enhanced antigen specific $\mathrm{T}$ cell responses and led to the rejection of moderately immunogenic murine tumors [26]. It was further reported that the mAb had a synergic effect in the treatment of cancer patients undergoing vaccines therapy. The anti-CTLA-4 antagonistic mAb in combination with a granulocyte-macrophage colony-stimulating factor (GM-CSF)-transduced cellular vaccine provided a synergistic effect in melanoma, mammary carcinoma, and prostate carcinoma models $[7,27]$. The combination of the CTLA- 4 blockade with other therapeutic strategies such as chemotherapy [28], radiotherapy [29], depletion of CD25+ Treg cells $[30,31]$ and CpG adjuvant [32] also exhibited enhanced anti-tumor activity and produced beneficial effects. Blockade of CTLA-4 concomitantly with inhibition of the other inhibitory signals and enhancement of co-stimulatory signals was reported to be significantly effective in promoting the rejection of melanomas [33, 34].

The development of ipilimumab (a humanized anti-CTLA-4 antagonistic mAb) has made it possible to treat patients with metastatic melanomas. Clinical studies, however, revealed significant autoimmune toxicities of the $\mathrm{mAb}$ in patients, such as diarrhea, bowel inflammation, and dermatitis [35, 36]. Guillain-Barré syndrome was also reported in a melanoma patient [37]. It is difficult to eliminate such a high degree of autoimmune toxicity associated with the CTLA-4 blockade, because there is no tumor specificity to the expression of CD80/CD86, furthermore, there is evidence that clinical responses might be associated with immune-related adverse events. It is thus essential to define a tolerated therapeutic window of the manipulation of CTLA-4 signaling for the development of tumor immunotherapy. In a study of 76 patients given ipilimumab with or without dacarbazine, the response rates were $17 \%$ and $5 \%$ in the combination group and the ipilimumab alone group, respectively [38]. When ipilimumab was administered to previously vaccinated metastatic melanoma and ovarian carcinoma patients, the mAb elicited extensive tumor necrosis with lymphocyte infiltrates without any serious toxicities [39, 40]. Further studies are needed to assess the optimal administration route and the clinical dose of ipilimumab. In addition, the most effective combination of CTLA-4 blockade with vaccination or other anti-tumor drugs has to be determined by defining biomarkers that predict clinical responses to the $\mathrm{mAb}$ therapy.

Table I. Experimental approaches targeting CD28 signaling pathway using antagonistic CTLA-4-Ig.

\begin{tabular}{lll}
\hline Approaches (clone) & condition & Reported functions [references] \\
\hline CTLA-4-Ig & autoimmune disease & Inhibited specific T cells activation [15] \\
CTLA-4-Ig & autoimmune disease & Inhibited specific T cells activation [12, 13]. Enhanced Treg function [14] \\
CTLA-4-Ig+Anti-CTLA-4 mAb (9H10) & transplantation & Inhibited donor T cells proliferation [20]. Depleted activated T cells [21] \\
CTLA-4-Ig & transplantation & Prolonged allograft survival [46] \\
\hline
\end{tabular}

Table 2. Experimental approaches targeting CTLA-4 signaling pathway using anti-CTLA-4 antagonistic mAbs.

\begin{tabular}{|c|c|c|}
\hline Approaches (clone) & condition & Reported functions [references] \\
\hline $\begin{array}{l}\text { Anti-CTLA-4 mAb (9H10)+ Anti-CTLA-4 mAb } \\
\text { (UC10-4F10-11) }\end{array}$ & tumor & Enhanced effector T cells function and inhibited Treg activation [27]. \\
\hline Anti-CTLA-4 mAb (9H10) + Radiotherapy & tumor & Increased survival [29]. \\
\hline Anti-CTLA- 4 mAb $(9 \mathrm{H} 10)+$ depletion of CD $25^{+}$Treg cells & tumor & Increased cytotoxic activity in T cells [30]. \\
\hline Anti-CTLA-4 mAb + depletion of CD25+ Treg cells & tumor & Enhanced specific cytotoxicity [31]. \\
\hline $\begin{array}{l}\text { Anti-CTLA- } 4 \text { mAb (UC10-4F10-11) + } \\
\text { CpG adjuvant }\end{array}$ & tumor & Enhanced specific $\mathrm{CD}^{+} \mathrm{T}$ cells function [32]. \\
\hline Anti-CTLA-4 mAb (9D9) + Anti-PD-1 mAb (RMP1-14) & tumor & Increased infiltrating T cells and reduced Tregs and myeloid cells [33]. \\
\hline Anti-CTLA-4 mAb (9D9) + Anti-41BB mAb (LOB12.3) & tumor & $\begin{array}{l}\text { Increased T cells infiltration, proliferation and cytokine production } \\
\text { [34]. }\end{array}$ \\
\hline MDX-010 & tumor & $\begin{array}{l}\text { Enhanced specific T cells function but induced autoimmune manifes- } \\
\text { tations }[36,37] \text {. }\end{array}$ \\
\hline MDX-010 + vaccination & tumor & Enhanced specific T cell function without serious toxicity $[39,40]$. \\
\hline Anti-CTLA-4 mAb (BNI3) & infection & Increased specific $\mathrm{CD} 4^{+} \mathrm{T}$ cell proliferation [44]. \\
\hline pCTLA-4-HBc & infection & Enhanced Th2 type function and accelerated virus cleanup [24]. \\
\hline
\end{tabular}

p, Plasmid. 
Since it is most likely that inhibitory signals in $\mathrm{T}$ cells play an essential part in the down-regulation of immune system during chronic infections, the role of CTLA-4 has been extensively studied in animal models and patients with infection. Whereas CTLA-4 appears not to be involved in the exhaustion of $\mathrm{CD} 8^{+} \mathrm{T}$ cells when mice are infected with viruses such as a lymphocytic choriomeningitis virus [41, 42], many reports demonstrate that CTLA-4 delivers inhibitory signals in $\mathrm{CD}^{+} \mathrm{T}$ cells during viral infections. In humans, CTLA-4 is up-regulated in HIV-specific CD4 ${ }^{+} \mathrm{T}$ cells during the acute HIV infection [43] and CTLA-4 blockade results in the proliferation of HIV-specific $\mathrm{CD}^{+} \mathrm{T}$ cells and a high level of cytokine secretion in vitro [44]. In addition, the level of soluble form of CTLA-4 (sCTLA-4) in the serum of chronic HBV patients is significantly higher than that in healthy individuals [45], suggesting that a high level of CTLA-4 expression in $\mathrm{T}$ cells is attributable to the impairment of $\mathrm{T}$ cell functions during chronic HBV infection. The findings clearly show that CLTA-4 blockade may serve as a means for the treatment of patients with acute and chronic viral infections. Taken together, antagonistic CTLA-4-Ig can be utilized to attenuate T cell functions in the treatment of autoimmunity and transplantation by blocking the engagement of CD28 with CD80/CD86, and anti-CTLA-4 antagonistic $\mathrm{mAbs}$ can be harnessed to enhance $\mathrm{T}$ cell functions in immunotherapies for malignancies and infections.

\section{Structure and function of PD-1}

PD-1 was initially identified in a T-cell hybridoma undergoing activation-induced cell death by subtractive hybridization [47]. Subsequent studies, however, have not shown a direct role for PD-1 in cell death. PD-1 is expressed during thymic development primarily on CD4-CD8- $T$ cells, and induced on peripheral $\mathrm{T}$ cells, $\mathrm{B}$ cells, and monocytes upon activation. The broader expression of PD-1 contrasts with restricted expression of other CD28 IgSF receptors to $\mathrm{T}$ cells, implying nonredundant roles for PD-1 molecule. This receptor is a $50-55 \mathrm{kDa}$ type I transmembrane glycoprotein and a member of IgSF that contains a single $\mathrm{V}$-set domain in its extracellular domain. Because PD-1 lacks a cystein residue that allows the molecule to covalently homodimerize, this receptor exists on the cell surface as a monomer or a noncovalantly associated oligomer.

The PD-1 cytoplasmic domain contains two tyrosine residues, with the membrane proximal one located with an ITIM motif and the other within an immunoreceptor tyrosine-based switch motif (ITSM). Whereas both tyrosine residues are phosphorylated following engagement with the ligand, mutagenesis studies indicate that only the membrane-distal tyrosine within the ITSM motif is required for the inhibitory activity of PD-1, as opposed to the tyrosine residue in the ITIM motif that is more typically associated with attenuation of antigen receptor signaling in other CD28 IgSF receptor molecules. In T cells, the phosphorylated ITSM recruits both Src homology region 2 domain-containing phosphatase-1 (SHP-1) and SHP-2.

The ligands for PD-1 are the CD80/CD86 IgSF members PD-L1 (PD-L1, also known as B7-H1, CD274) and PD-L2 (B7-DC, CD273). Both ligands are type I glycoproteins with tandemly alligned $\mathrm{V}$-set and C1-set extracellular domains. PD-L1 is expressed on lymphoid cells such as T and B cells as well as nonlymphoid organs including heart, liver, lung, pancreas, muscle, and placenta, suggesting that PD-L1 may regulate self-reactive $\mathrm{T}$ or $\mathrm{B}$ cells in peripheral sites and may regulate inflammatory responses in the target organs. In contrast, PD-L2 expression is restricted to DCs and macropahges, suggesting nonredundant roles of PD-L1 and PD-L2 in immune responses.

Because both PD-1 and PD-L1 extracellular domain preparations exist as monomers in solution, the binding mode is expected to be different from that of CTLA-4/CD80/CD86. Based on X-ray analysis, PD-1/PD-L1 complex is a 1:1 complex of monomeric PD-1 and PD-L1 in the crystal. The complex has a buried surface area $\left(1,870 \AA^{2}\right)$, which is significantly larger than that of CTLA-4/CD80 $\left(1,200 \AA^{2}\right)$. It is noteworthy that the Ig variable domains of the PD-1/PD-L1 complex are similar to those of $\mathrm{T}$ cell antigen receptors and Abs, raising a possibility that the loops of the PD-1/PD-L1 complex may bind another molecule.

The disruption of a gene encoding PD-1 causes autoimmune diseases, like a late-onset, progressive arthritis and lupus-like glomerulonephritis in mice on the C57Bl/ 6 background and a dilated cardiomyopathy in Balb/c mice, PD-1, hence, is considered to be involved in the maintenance of peripheral tolerance. In addition, PD-1-deficient mice are extremely sensitive to murine hepatitis virus strain-3 infection [48-50]. PD-1 is up-regulated in Tregs when infected with feline immunodeficiency virus [51] and in T cells of chronic hepatitis B virus patients, compared to healthy individuals and acute hepatitis B patients [52], thereby PD-1 may play a critical role in the impairment of $\mathrm{T}$ cell functions. It is worthy of note that $\mathrm{HCV}$-infected individuals whose $\mathrm{CD} 4^{+} \mathrm{T}$ cells express a high level of PD-1 exhibit a poor response to HBV vaccine, unless the PD-1 signaling is blocked [53]. Up-regulation of PD-1 correlates with the prognosis of HCC (hepatic cell carcinoma) patients and raises the rate of recurrence $[54,55]$. A recent research demon- 
strated enhanced PD-1 expression in actinic cheilitis and oral squamous cell carcinoma [56]. It is highly likely that the impairment of tumor-infiltrating $\mathrm{T}$ lymphocyte functions is due to the expression of PD-1 on the effector cells [57]. Evidence is accumulating that the PD-1/PD-L1/PD-L2 pathway plays a key role in autoimmune diseases and tumor evasion.

\section{Therapeutic application of PD-1}

In various ethnic groups, PD-1 gene haplotype appears to be associated with susceptibility to autoimmune diseases such as systemic lupus erythematosus [58], rheumatoid arthritis [59], and type I diabetes [60]. To develop immunotherapies for autoimmune diseases, the manipulation of PD-1/PD-L pathway has been investigated in animal models (Table 3). The injection of adenovirus harboring cDNA encoding PD-L1 into lupus-prone mice partially ameliorated the development of nephritis [61]. The transfer of embryonic stem cell-derived DCs expressing myelin oligodendrocyte glycoprotein (MOG) peptide reduced T cell responses to MOG, cell infiltration into spinal cord, and the severity of experimental autoimmune encephalomyelitis (EAE) [62]. IFN- $\beta$ used for the treatment of multiple sclerosis has been shown to up-regulate PD-L1 on monocytes and DCs, suggesting that inhibitory signals through the PD-1/PD-L pathway contribute to the anti-inflammatory effect [63].

On the contrary, the blockade of PD-1/PD-L signaling is expected to potentiate $\mathrm{T}$ cell effector functions. Whereas the immunization of mice with lentivector encoding tumor antigens markedly increases the infiltration of antigen-specific $\mathrm{CD} 8^{+} \mathrm{T}$ cells and $\mathrm{CD}^{+} \mathrm{T}$ cells and generates Ag-specific antitumor effects, it also induces the expression of PD-L1 in the tumor lesions and PD-1 in the tumor-infiltrating CD8 ${ }^{+}$ cells, limiting the anti-tumor effects of lentivector immunization. Blocking PD-1/PD-L pathway is, however, shown to rescue the effector functions of the $\mathrm{CD} 8{ }^{+} \mathrm{T}$ cells and enhance the antitumor efficacy of lentivector immunization [64, 65]. In addition, the blockade of PD-1/PD-L pathway using a fully humanized anti-PD-1 antagonistic mAb increases the numbers and functions of tumor-specific $\mathrm{T}$ cells and promotes cytokine production in humans [66]. Furthermore, retroviral small interfering RNA (siRNA) delivery has been proven to reduce surface PD-1 expression and improve murine as well as human $\mathrm{T}$ cell immune functions, demonstrating that blocking PD-1/PD-L pathway is a promising approach to achieve immunopotentiation in tumor therapy [67, 68].

In recent clinical trials of anti-PD-1 and anti-PD-L1 antagonistic mAbs, objective and durable tumor responses were observed in patients with advanced cancers, including non-small-cell lung cancer, melanoma, and renal cell carcinoma [69, 70]. The grade 3 or 4 drug-related adverse events occurred in only $14 \%$ and $9 \%$ of patients who underwent anti-PD-1 and anti-PD-L1 treatment, respectively. The incidence was significantly less than that for anti-CTLA- $4 \mathrm{mAb}$ treatment, in which inflammatory or autoimmune toxic effects were observed in $20 \%-30 \%$ of patients [71]. This is probably because the PD-1/PD-L1 pathway predominantly regulates the effector phase of $\mathrm{T}$ cell responses against tumor cells. The blockade of PD-1/PD-L1 engagement is thus one of the most promising immunotherapies for cancer in future.

Since the attenuation of $\mathrm{T}$ cell effector functions by PD-1/PD-L pathway has been suggested in various infectious diseases, the disruption of the inhibitory pathway may be a promising means to enhance immune responses in patients with infections. PD- 1 is highly expressed in T cells when individuals are infected with viruses such as HIV, HBV, and HCV. Blocking the PD-1/PD-L1 pathway promotes HIV-specific $\mathrm{CD}^{+}$and $\mathrm{CD} 8^{+} \mathrm{T}$ cell proliferation and augments the secretion of anti-viral cytokines [72]. Anti-PD-L1 mAb decreases the level of PD-1 expression and promoted IL-12 production as well as STAT-1 activation in monocytes/macrophages derived from $\mathrm{HCV}$-infected individuals [73]. It is worthy of note that exhausted $\mathrm{CD} 8^{+} \mathrm{T}$ cells during viral infections express multiple inhibitory receptors and blocking PD-1 and LAG-3 (lymphocyte-activation gene 3 ) synergistically improved $\mathrm{T}$ cell responses and reduced viral load [74]. Furthermore, PD-L1 blockade leads to the enhancement of $\mathrm{CD}^{+} \mathrm{T}$ cell proliferation in Helicobacter pylori infection and Schistosoma mansoni infection [75, 76]. In an indirect acute lung injury model, PD-1-deficient mice show a higher survival rate [77], and blocking PD-1/PD-L pathway in migratory langerhans cells and DCs can enhance $\mathrm{T}$ cell activation [78].

The manipulation of the inhibitory PD-1/PD-L1 signaling is expected to be an effective means for maintaining grafts in transplantation. Several studies show that forced expression of PD-L1 in grafted cells decreases the incidence of allograft rejection [79] and GVHD lethality [80] in animal models. The combination of antagonistic PD-L1-Ig and anti-CD154 mAb or anti-ICOS antagonistic mAb prolongs cardiac allograft survival, which is accompanied by reduced intragraft expression of IFN- $\gamma$ and IFN- $\gamma$-induced chemokines [79]. Similarly, PD-L1-Ig synergizes with anti-CD154 $\mathrm{mAb}$ in promoting long-term survival of islet allografts [81]. 
Table 3. Immunotherapeutic approaches manipulating PD-I/PD-L pathway using gene transfer and/or antagonistic mAbs.

\begin{tabular}{|c|c|c|}
\hline Approaches (clone) & Condition & Reported functions [references] \\
\hline PD-L1 transfection & Autoimmune disease & Partially prevented nephritis [61]. \\
\hline PD-L1-highly- expressing DCs transfection & Autoimmune disease & Prevented EAE [62]. \\
\hline IFN- $\beta$ & Autoimmune disease & Up-regulated PD-L1 level and prevented inflammation [63]. \\
\hline $\begin{array}{l}\text { Anti-PD-1 mAb (RMPI-14) + Anti-PD-L1 mAb } \\
\text { (10F.9G2) + lentivector immunization }\end{array}$ & Tumor & Enhanced anti-tumor effects $\left[64,65^{*}\right]$. \\
\hline Anti-PD-1 mAb (anti-Hpd-1.5) & Tumor & Increased specific T cell proliferation and cytokine production [66]. \\
\hline siRNA & Tumor & Down-regulated PD-1 level and enhanced T cell functions $[67,68]$. \\
\hline Anti-PD-1 mAb & Timor & Objective and durable tumor responses in a clinical study [69]. \\
\hline Anti-PD-L1 mAb & Tumor & Objective and durabole tumor responses in a clinical study [70]. \\
\hline Anti-PD-1 mAb & Infection & Increased specific T cell proliferation and cytokine production[ $\left[72^{*}\right]$. \\
\hline PD-1 KO & Infection & Improved survival rate [77]. \\
\hline $\begin{array}{l}\text { Anti-PD-1 mAb (EH12) + Anti-PD-L1 mAb } \\
\text { (29E.2A3) + Anti-PD-L2 mAb (24F.10C12) }\end{array}$ & Infection & Enhanced T cells functions [78]. \\
\hline Anti-PD-L1 mAb (M1H1) & Infection & Increased IL-12 production and enhanced STAT-1 activation [73]. \\
\hline $\begin{array}{l}\text { Anti-PD-L1 mAb (10F.9G2) + Anti-LAG-3 mAb } \\
\text { (C9B7W) }\end{array}$ & Infection & Enhanced T cell functions and reduced virus load [74]. \\
\hline Anti-PD-L1 mAb (MIH5) & Infection & Increased $\mathrm{CD}^{+} \mathrm{T}$ cell proliferation $\left[75^{*}, 76\right]$ \\
\hline PD-L1-Ig + anti-CD154 mAb (MR1) & Transplantation & Prolonged allograft survival [77*-81]. \\
\hline $\begin{array}{l}\text { Anti-PD-1 mAb (J43) + Anti-CTLA-4 mAb } \\
(\mathrm{C} 10-4 \mathrm{~F} 10-1)\end{array}$ & Transplantation & Prevented GVHD [80]. \\
\hline
\end{tabular}

$\mathrm{KO}$, Knock-out; ${ }^{\text {, }}$ mAb clone numbers were not given.

\section{Structure and function of BTLA}

BTLA (B and T cell attenuator, CD272) is a member of IgSF family and a $32 \mathrm{kDa}$ type I transmembrane glycoprotein consisting of an I-set (intermediate-set) extracellular domain, a transmembrane region, and a cytoplasmic region, suggesting that this molecule is distinct from the CD28 family [82]. Because BTLA lacks a cystein residue needed for dimerization, it is likely to exist as a monomer on the cell surface. The presence of two ITIM motifs and an ITSM motif in its cytoplasmic domain indicates that this molecule functions as an inhibitory receptor [83]. Cross-linking BTLA with agonistic mAbs stimulates its tyrosine phosphorization and leads to SHP-1 and SHP-2 recruitment, providing a mechanism for BTLA-mediated signal inhibition. Identified initially as a molecule selectively expressed on Th1 cells, it is induced on $\mathrm{T}$ cells during activation and remains expressed more strongly on polarized Th1, not Th2 cells [84], implying that BTLA may specifically down-regulate Th1-mediated inflammatory responses [85]. In fact, several studies demonstrate that signaling through BTLA attenuates $\mathrm{T}$ lymphocyte proliferation [86-88]. In addition, BTLA gene polymorphisms may link to the development of rheumatoid arthritis, malignant breast cancer [89-92].

BTLA binds herpes virus entry mediator
(HVEM), a member of tumor necrosis factor receptor superfamily (TNFRSF). This interaction is unusual in that it represents the first example of a TCFRSF functioning as a ligand. Based on X-ray crystal structure of BTLA/HVEM complex, a single globular BTLA interacts with the membrane distal region of rod-shaped HVEM. Compared to the CTLA-4/CD80 binding site, BTLA uses a distinct surface to interact with HVEM. HVEM-deficient mice show enhanced T cell proliferation and $\mathrm{CD}^{+} \mathrm{T}$ cell-dependent proinflammatory cytokine production in response to concanavalin $\mathrm{A}$ stimulation [93].

\section{Therapeutic application of BTLA}

BTLA/HVEM pathway plays an important role in the maintenance of immune tolerance and the prevention of autoimmune diseases (Table 4). BTLA-deficient mice develop rheumatoid arthritis [94], lymphocytic infiltration, autoimmune hepatitis (AIH)-like diseases, and EAE [95, 96]. HVEM-deficient mice show increased susceptibility to MOG peptide-induced EAE and increased T cell proliferation and cytokine production [93]. Antagonistic HVEM-Ig aggravates autoimmunity in collagen-induced arthritis on DBA1 background mice [97]. Thus, the forced expression of BTLA in activated T cells would be a promising strategy for the treatment of autoimmune diseases. 
Table 4. Experimental approaches targeting BTLA/HVEM pathway using antagonistic HVEM-lg and anti-BTLA mAbs.

\begin{tabular}{lll}
\hline Approaches (clone) & Condition & Reported functions [references] \\
\hline HVEM KO & Autoimmune disease & Increased T cell proliferation and cytokine production [93] \\
HVEM-Ig & Autoimmune disease & Increased T cell proliferation [97] \\
Vaccination + CpG adjuvant & Tumor & Down-regulated BTLA level and decreased BTLA-HVEM-mediated inhibition \\
& & [98] \\
Anti-BTLA mAb (6A6) & Infection & Reduced the incidence of cerebral malaria [99] \\
Anti-BTLA mAb & Transplantation & Rejected MHC class II-mismatched cardiac allografts [100] \\
Anti-BTLA mAb (6F7) + CTLA-Ig Transplantation & Prolonged allograft survival [102] \\
\hline
\end{tabular}

$\mathrm{s}$, Soluble; *, the mAbs clone numbers were not given.

Regarding tumor immunity, tumor antigen-specific $\mathrm{CD}^{+} \mathrm{T}$ cells appear to persistently express BTLA. It has been reported that $\mathrm{CpG}$ vaccination partially down-regulates the expression of BTLA in tumor antigen-specific $\mathrm{CD} 8^{+} \mathrm{T}$ cells and blocks the BTLA/HVEM-mediated inhibitory signal [98]. Although blocking the BTLA/HVEM pathway seems to be relevant as a means to enhance effector T cell functions, careful attention should be paid to the complexity of HVEM-interacting molecules. CD160, an IgSF inhibitory receptor, also binds HVEM. In addition, LIGHT, a TNF family member, delivers a costimulatory signal upon engagement with HVEM. These multiple pathways make it difficult for us to establish novel therapeutic interventions for malignancies.

The manipulation of BTLA/HVEM pathway may become a promising strategy to treat patients with infections. BTLA is induced during $P$. berghei ANKA infection in mice and anti-BTLA antagonistic $\mathrm{mAb}$ significantly reduces the incidence of cerebral malaria caused by the protozoa [99]. Thus, pathogens pertubing the BTLA/HVEM pathway may represent ideal targets for anti-BTLA mAb immunotherapy.

In transplantation, the BTLA/HVEM pathway has a unique role in regulating allogeneic responses. It is noteworthy that BTLA, not PD-1, is strongly induced in alloreactive $\mathrm{T}$ cells from mice transplanted with partially MHC-mismatched cardiac allografts. Whereas the allografts survive relatively long term in wild type mice, a rapid rejection is observed in BTLA-deficient mice in this partially mismatched model [100]. This indicates that BTLA and PD-1 may play nonredundant roles in transplantation. BTLA seems to be dominant over PD-1 when immune responses are relatively weak, while PD-1 plays a major role in strong allo-responses. Soluble HVEM-Ig or anti-HVEM mAb can prevent GVHD and allograft rejection [101]. The combination of antagonistic anti-BTLA mAb and CTLA-4-Ig prolongs allograft sur- vival, whereas CTLA-4-Ig or anti-BTLA mAb alone fails to prevent graft rejection [102].

\section{Summary}

Inhibitory receptors, CTLA-4, PD-1, and BTLA deliver negative signals that play an important role in regulating $\mathrm{T}$ cell activation and maintaining peripheral tolerance. Manipulations of negative signals mediated by the inhibitory receptors and/or the positive signals mediated by co-stimulatory receptors may provide therapeutic strategies for autoimmune diseases, malignancies, infectious diseases, and transplantation. Some therapeutic reagents are now being tested in clinical trials (Table 5). Major international pharmaceutical companies are sponsoring a plethora of clinical trials on the modulation of inhibitory signaling pathways in various diseases such as rheumatoid arthritis, ulcerative colitis, melanoma, HCV infection, renal transplantation.

In order to achieve effective control of inhibitory signaling pathways as therapeutic interventions, appropriate biomarkers should be developed to identify which inhibitory pathways dominate in the particular diseases, because different inhibitory pathways may be involved in the onset of different diseases. It is also essential to develop combinatorial therapies using two or more therapeutic agents, when multiple inhibitory signals participate in the diseases. Because the inhibitory signaling is generally initiated by the interaction between cell surface receptors and ligands, the blockade of the ligation can be achieved simply by humanized mAbs or recombinant extracellular domains of the molecules. Anti-CTLA-4 mAb is the first immunomodulator approved by US Food and Drug Administration, and encouraging clinical findings have been reported for mAbs specific for the PD-1/PD-L pathway. Novel immunotherapies using these agents are especially promising in the treatment of patients with malignancies in the near future. 
Table 5. Therapeutics targeting inhibitory receptor/ligands being tested in clinical trials.

\begin{tabular}{|c|c|c|}
\hline Treatment & Other name & Conditions(Phase) \\
\hline \multirow[t]{2}{*}{ CTLA-4Ig } & Abatacept & $\begin{array}{l}\text { Type } 1 \text { diabetes mellitus (II), psoriasis vulgaris (I+II), lupus nephritis (II), lupus erythematosus, sys- } \\
\text { temic (II), rheumatoid arthritis (I+II), Wegener's granulomatosis (II+III), Takayasu's arteritis (I+II), giant } \\
\text { cell arteritis (I+II), multiple sclerosis (II), allergic asthma (II). }\end{array}$ \\
\hline & Belatacept & Rheumatoid arthritis (I+II+III) \\
\hline (CTLA-4 IgG4m) & RG2077 & Lupus erythematosus, systemic (I+II), lupus nephritis (I+II). \\
\hline \multirow[t]{2}{*}{ Anti-CTLA-4 } & $\begin{array}{l}\text { MDX-010/ } \\
\text { Ipilimumab }\end{array}$ & $\begin{array}{l}\text { Synovial Sarcoma (II), wilm's tumor }(\mathrm{I}) \text {, lymphoma (I), neuroblastoma (I), melanoma (I+III), malignant } \\
\text { fibrous histiocytoma of bone (I), kidney cancer (I), prostate cancer (I), high risk stage III melanoma (III), } \\
\text { leukemia (I), lung Cancer (I), myelodysplastic syndromes (I), ovarian cancer (I), pancreatic cancer (II), } \\
\text { extensive stage small cell lung cancer (II). }\end{array}$ \\
\hline & $\begin{array}{l}\text { CD-675,206/ } \\
\text { Tremelimumab }\end{array}$ & $\begin{array}{l}\text { Melanoma (II), prostatic neoplasms (I), hepatocellular carcinoma (I), hepatitis C virus chronic infection } \\
\text { (I), bladder cancer (I), renal cell carcinoma (II). }\end{array}$ \\
\hline \multirow[t]{2}{*}{ Anti-CD80 } & IDEC-114 & Non-Hodgkin's lymphoma (I+II). \\
\hline & Galiximab & Non-Hodgkin's lymphoma (I+III). \\
\hline \multirow[t]{4}{*}{ Anti-PD-1 } & MDX1106 & melanoma $(\mathrm{I})$, hepatitis $\mathrm{C}(\mathrm{I})$. \\
\hline & CT-011 & $\begin{array}{l}\text { Prostatic neoplasms (II), breast cancer(I), colon cancer (I), pancreatic cancer (I), sarcoma (I), ovarian } \\
\text { cancer (I), renal cell carcinoma (II), acute myelogenous leukemia (II), multiple myeloma (II). }\end{array}$ \\
\hline & BMS-936558 & Renal cell carcinoma (I), non-small cell lung cancer (I). \\
\hline & ONO-4538 & malignant solid tumor (I). \\
\hline Anti-PD-L1 & BMS-936559 & $\begin{array}{l}\text { Stage III or IV melanoma, non-Hodgkin's lymphoma (I), Hodgkin Lymphoma (I), multiple myeloma (I), } \\
\text { chronic myelogenous leukemia (I). }\end{array}$ \\
\hline Anti-PD-L2 & rHIgM12B7 & Melanoma (I). \\
\hline
\end{tabular}

This information was obtained from clinicaltrails.gov.

\section{Abbreviations}

CTLA-4: cytotoxic T lymphocyte associated antigen-4; PD-1: programmed death-1; PD-L1: programmed death-ligand1; BTLA: B and T cell attenuator; TCR: T cell receptor; CD: cluster of differentiation; APCs: antigen presenting cells; ICOS: inducible costimulator; ICOS-L: inducible costimulator-ligand; mAbs: monoclonal antibodies; V-set: variable domain like-set; C1-set: constant1-set; I-set: intermediate-set; IgSF: immunoglobulin superfamily; ITIM: immunoreceptor tyrosine-based inhibitory motif; ITSM: immunoreceptor tyrosine-based switch motif; APC: antigen presenting cell; Tregs: $\mathrm{T}$ regulatory cells; DCs: dendritic cells; Ig: immunoglobulin; RA: rheumatoid arthritis; EAE: experimental allergic encephalitis; GVHD: graft-versus-host diseases; IFN: interferon; IL-4: interleukin-4; GM-CSF: ranulocyte macrophage-colony stimulating factor; HIV: human immunodeficiency virus; $\mathrm{HBV}$ : hepatitis B virus; $\mathrm{HCV}$ : hepatitis $\mathrm{C}$ virus; HCC: hepatic cell carcinoma; $\mathrm{CDNA}$ : complementary deoxyribonucleic acid; MOG: myelin oligodendrocyte glycoprotein; siRNA: small interfering ribonucleic acid; STAT-1: Signal transducers and activators of transcription; LAG-3: lymphocyte-activation gene 3; SHP: src homology 2-containing tyrosine phosphatase; HVEM: herpes virus entry mediator; TNFRSF: tumor necrosis factor receptor superfamily; AIH: autoimmune hepatitis;
LIGHT: homologous to lymphotoxins, shows inducible expression, and competes with herpes simplex virus glycoprotein $\mathrm{D}$ for herpes virus entry mediator, a receptor expressed by T lymphocytes; MHC: major histocompatibility complex.

\section{Acknowledgments}

We gratefully acknowledge the financial support from the Public Technology Research and Social Development Project of Zhejiang Province (20011C23004) and Zhejiang Provincial Natural Science Foundation of China (LY12B02019).

\section{Competing Interests}

The authors have declared that no competing interest exists.

\section{References}

1. Schwartz RH. T cell anergy. Annu Rev Immunol. 2003; 21: 305-334.

2. Chen L. Immunological ignorance of silent antigens as an explanation of tumor evasion. Immunol Today. 1998; 19: 27-30.

3. Chambers CA, Kuhns MS, Egen JG, et al. CTLA-4-mediated inhibition of $\mathrm{T}$ cell responses: mechanisms and manipulation in tumor immunotherapy. Annu Rev Immunol. 2001; 19: 565-594.

4. Jain N, Nguyen H, Chambers C, et al. Dual function of CTLA-4 in regulatory $\mathrm{T}$ cells and conventional $\mathrm{T}$ cells to prevent multiorgan autoimmunity. PANS. 2010; 107: 1524-1528.

5. Walunas TL, Bakker CY, Bluestone JA. CTLA-4 ligation blocks CD-28-dependent T cell activation. J Exp Med. 1996; 183: 2541-2550.

6. Walunas TL, Lenschow DJ, Bakker CY, et al. CTLA-4 can function as a negative regulator of T cell activation. J Immunol. 1994; 1: 405-413.

7. Chambers CA, Kuhns MS, Egen JG, et al. CTLA-4-mediated inhibition in regulation of $\mathrm{T}$ cell responses: mechanisms and manipulation in tumor immunotherapy. Annu Rev Immunol, 2001; 19: 565-594. 
8. Rudd CE, Schneider H. Unifying concepts in CD28, ICOS and CTLA-4 co-receptor signaling. Nature Rev Immunol. 2003; 3: 544-556.

9. Salomon B, Bluestone JA. Complexities of CD28/B7: CTLA-4 co-stimulatory pathways in autoimmunity and transplantation. Annu Rev Immunol. 2001; 19: 225-252.

10. Hori S, Takahashi T, Sakaguchi S. Control of autoimmunity by naturally arising regulatory CD4 ${ }^{+} \mathrm{T}$ cells. Adv Immunol. 2003; 81: 331-371.

11. Wing K, Onishi Y, Prieto-Martin P, et al. CTLA-4 control over Foxp3 ${ }^{+}$ regulatory $\mathrm{T}$ cell function. Science 2008; 322: 271-275.

12. Cross AH, Girard TJ, Giacoletto KS, et al. Long-term inhibition of murine experimental autoimmune encephalomyelitis using CTLA-4-Fc supports a key role for CD28 costimulation. J Clin Invest. 1995; 95: 2783-2789.

13. Knoerzer DB, Karr RW, Schwartz BD, et al. Collagen-induced arthritis in the BB rat. Prevention of disease by treatment with CTLA-4-Ig. J Clin Invest. 1995; 96: 987-993.

14. Kremer JM, Genant HK, Moreland LW, et al. Effects of abatacept in patients with methotrexate-resistant active rheumatoid arthritis: a randomized trial. Ann Intern Med. 2006; 144: 865-876.

15. Finck BK, Linsley PS, Wofsy D. Treatment of murine lupus with CTLA4Ig. Science. 1994; 265: 1225-1227.

16. Laskowski IA, Pratschke J, Wilhelm MJ, et al. Anti-CD28 monoclonal antibody therapy prevents chronic rejection of renal allografts in rat. J Am Soc Nephrol. 2002; 13: 519-527.

17. Dong VM, Yuan X, Coito AJ, et al. Mechanisms of targeting CD28 by a signaling monoclonal antibody in acute and chronic allografts rejection. Transplantation. 2002; 73: 1310-1317.

18. Guillonneau C, Séveno C, Dugast AS, et al. Anti-cd28 antibodies modify regulatory mechanisms and reinforce tolerance in CD40Ig-treated heart allograft recipients. J Immunol. 2007; 179: 8164-8171.

19. Urakami H, Ostanin DV, Hunig T, et al. Combination of donor-specific blood transfusion with anti-CD28 antibody synergizes to prolong graft survival in rat liver transplantation. Transplant Proc. 2006; 38: 3244-3246.

20. Yu XZ, Bidwell SJ, Martin PJ, et al. CD28-specific antibody prevents graft-versus-host disease in mice. J Immunol. 2000; 164: 4564-4568.

21. Yu XZ, Albert MH, Martin PJ, et al. CD28 ligation induces transplantation tolerance by IFN-gamma-dependent depletion of T cells that recognize alloantigens. J Clin Invest. 2004; 113: 1624-1630.

22. Dengler TI, Szabo G, Sido B, et al. Prolonged allograft survival but no tolerance infuction by modulation CD28 antibody JJ319 after high-responder rat heart transplantation. Transplantation. 1999; 67: 392-398.

23. Pearson TC, Alexander DZ, Winn KJ, et al. Transplantation tolerance induced by CTLA-4-Ig. Transplantation. 1994; 57: 1701-1706.

24. Zhai C, Yu L, Zhu H, et al. Porcine CTLA-4-Ig prolong islet xenografts in rats by downregulating the direct pathway of T-cell activation. Xenotransplantation. 2011; 18: 40-45.

25. Álvarez-Quiroga C, Abud-Mendoza C, Doníz-Padilla L, et al. CTLA-4-Ig therapy diminishes the frequency but enhances the function of Treg cells in patients with Rheumatoid Arthritis. J. Clin. Immunol. 2011; 31: 588-595.

26. Leach DR, Krummel MF, Allison JP. Enhancement of antitumor immunity by CTLA-4 blockade. Science. 1996; 271: 1734-1736.

27. Peggs KS, Quezada SA, Korma AJ, et al. Principles and use of anti-CTLA-4 antibody in human cancer immunothearpy. Curr Opin Immunol. 2006; 18: 206-213.

28. Peggs KS, Quezada SA, Chambers CA, et al. Blockade of CTLA-4 on both effector and regulatory $\mathrm{T}$ cell compartments contributes to the antitumor anctivity of anti-CTLA-4 antibodies. J Exp Med. 2009; 206: 1717-1725.

29. Mokyr MB, Kalinichenko T, Gorelik L, et al. Realization of the therapeutic potential of CTLA-4 blockade in low-dose chemotherapy-treated tumor-beating mice. Cancer Res. 1998; 58: 5301-5304.

30. Demaria S, Kawashima N, Yang AM, et al. Immune-mediated inhibition of metastases after treatment with local radiation and CTLA-4 blockade in a mouse model of breast cancer. Clin Cancer Res. 2005; 11: 728-734.

31. Sutmuller RPM, van Duivenvoorde LM, van Elsas A, et al. Synergism of cytotoxic $\mathrm{T}$ lymphocyte-associated $\mathrm{T}$ cells in antitumor therapy reveals alternative pathways for suppression of autoreactive cytotoxic $\mathrm{T}$ lymphocyte responses. J Exp Med. 2001; 194: 823-832.

32. Suarez N, Alfaro C, Dubrot J, et al. Synergistic effects of CTLA-4 blockade with tremelimumab and elimination of regulatory $\mathrm{T}$ lymphocytes in vitro and in vivo. Int J Cancer. 2011; 129: 374-386.

33. Davila E, Kennedy R, Celis E. Generation of antitumor immunity by cytotoxic $\mathrm{T}$ lymphocyte epitope peptide vaccination, CpG-oligodeoxynucleotide adjuvant, and CTLA-4 blockade. Cancer Res. 2003; 63: 3281-3288.
34. Curran MA, Montalvo W, Yagita H, et al. PD-1 and CTLA-4 combination blockade expands infiltrating $\mathrm{T}$ cells and reduces regulatory $\mathrm{T}$ and myeloid cells within B16 melanoma tumors. PANS. 2010; 107: 4275-4280.

35. Curran MA, Kim M, Montalvo W, et al. Combination CTLA-4 blockade and 4-1BB activation enhances tumor rejection by increasing $\mathrm{T}$ cell infiltration, proliferation and cytokine production. PLoS ONE. 2011; 6: 1-11.

36. Sanderson $\mathrm{K}$, Scotland $\mathrm{R}$, Lee $\mathrm{P}$, et al. Autoimmunity in a phase I trial of a fully human anti-CTLA-4 monoclonal antibody with multiple peptides and Montanide ISA 51 for patients with resected stages III/IV melanoma. J Clin Oncol. 2005; 23: 741-750.

37. Phan GQ, Yang JC, Aherry RM, et al. Cancer regression and autoimmunity induced by cytotoxic $\mathrm{T}$ lymphocyte-associated antigen antigen 4 blockade in patients with metastatic melanoma. Proc Natl Acad Sci. 2003; 100: 8371-8377.

38. Wilgenhof S, Neyns B. Anti-CTLA-4 antibody-induced Guillain-Barré syndrome in a melanoma patient. Ann Oncol. 2011; 22: 991-993.

39. Hersh EM, Weber J, Powderly J, et al. A phase II, randomized multi-center study of MDX-010 alone or in combination with dacarbazine (DTIC) in stage IV metastatic malignant melanoma. J Clin Oncol. 2004; 22: s7511.

40. Hodi FS, Mihm MC, Soiffer RJ, et al. Biologic activity of cytotoxic T lymphocyte-associated antigen 4 antibody blockade in previously vaccinated metastatic melanoma and ovarian carcinoma patients. Pron Natl Acad Sci. 2003; 100: 4712-4717.

41. Yuan J, Ginsberg B, Page D, et al. CTLA-4 blockade increases antigen-specific $\mathrm{CD}^{+} \mathrm{T}$ cells in prevaccinated patients with melanoma: three cases. Cancer Immunol Immunother. 2011; 60: 1137-1146.

42. Bachmann MF, Waterhouse $\mathrm{P}$, Speiser DE, et al. Normal responsiveness of CTLA-4-deficient anti-viral cytotoxic T cells. J Immunol. 1998; 160: 95-100.

43. Barber DL, Wherry EJ, Masopust D, et al. Restoring function in exhausted CD8 T cells during chronic viral infection. Nature. 2006; 439: 682-687.

44. Leng Q, Bentwich Z, Magen E, et al. CTLA-4 upregulated during HIV infection: association with anergy and possible target for therapeutic intervention. Aids. 2002; 16: 519-529.

45. Kaufmann DE, Kavanagh DG, Pereyra F, et al. Upregulation of CTLA-4 by HIV-specific $\mathrm{CD}^{+}{ }^{+} \mathrm{T}$ cells correlates with disease progression and defines a reversible immune dysfunction. Nat Immunol. 2007; 8: 1246-1254.

46. Cao J, Zhang L, Huang S, et al. Aberrant production of soluble co-stimulatory molecules CTLA-4 and CD28 in patients with chronic hepatitis B. Microb Pathog. 2011; 51: 262-267.

47. Ishida Y, Agata Y, Shibahara K, et al. Induced expression of PD-1, a novel member of the immunoglobulin gene superfamily, upon programmed cell death. EMBO J.1992; 11: 3887-3895.

48. Nishimura H, Nose M, Hiai H, et al. Develpoment of lupus-like autoimmune diseases by disruption of the PD-1 gene encoding an ITIM motif-carring immunoreceptor. Immunity. 1999; 11: 141-151.

49. Nishimura H, Okazaki T, Tanaka Y, et al. Autoimmune dilated cardiomyopathy in PD-1 receptor-deficient mice. Science. 2001; 291: 319-322.

50. Chen Y, Wu S, Guo G, et al. Programmed death (PD)-1-dificient mice are extremely sentitive to murine hepatitis virus strain-3 (MHV-3) infection. PLoS Pathog. 2011; 7: e1001347.

51. Achleiner A, Clark ME, Bienzle D. T-regulatory cells infected with immunodeficiency virus up-regulate programmed death-1(PD-1). Vet Immunol Immunopathol. 2011; 143: 307-313.

52. Chen J, Wang XM, Wu XJ, et al. Intrahepatic levels of PD-1/PD-L1 correlate with liver inflammation in chronic hepatitis B. Inflamm Res. 2011; 60: 47-53.

53. Moorman JP, Zhang CL, Ni L, et al. Impaired hepatitis B vaccine responses during chronic hepatitis $\mathrm{C}$ infection: Involvement of the PD-1 pathway in regulating $\mathrm{CD}^{+} \mathrm{T}$ cell responses. Vaccine. 2011; 29: 3169-3176.

54. Zeng Z, Shi F, Zhou L, et al. Upregulation of circulating PD-L1/PD-1 is associated with poor post-cryoablation prognosis in patients with HBV-related hepatocellular carcinoma. PLoS One. 2011; 6(9): e23621.

55. Shi F, Shi M, Zeng Z, et al. PD-1 and PD-L1 upregulation promotes CD8 ${ }^{+}$ $\mathrm{T}$ cell apoptosis and postoperative recurrence in hepatocellular carcinoma patients. Int J Cancer. 2011; 128: 887-896.

56. Malaspina TS, Gasparoto TH, Costa MR, et al. Enhanced programmed death 1 (PD-1) and PD-1 ligand (PD-L1) expression in patients with actinic cheilitis and oral squamous cell carcinoma. Cancer Immunol Immunother. 2011; 60: 965-974.

57. Wang SF, Fouquet $S$, Chapon $M$, et al. Early $\mathrm{T}$ cell signalling is reversibly altered in PD-1+ $\mathrm{T}$ lymphocytes infiltrating human tumors. PLoS One. 2011; 6: e17621. 
58. Wang SC, Chen YJ, Ou TT, et al. Programmed Death-1 Gene Polymorphisms in Patients With Systemic Lupus Erythematosus in Taiwan. J Clin Immunol. 2006; 26: 506-511.

59. Raptopoulou AP, Bertsias G, Makrygiannakis D, et al. The Programmed Death1/Programmed Death Ligand 1 Inhibitory Pathway Is Up-Regulated in Rheumatoid Synovium and Regulates Peripheral T Cell Responses in Human and Murine Arthritis. Arthritis Rheum. 2010; 62: 1870-1880.

60. Ni R, Ihara K, Miyako K, et al. PD-1 gene haplotype is associated with the development of type diabetes I mellitus in Japanese children. Hum Genet. 2007; 121: 223-232.

61. Ding $\mathrm{H}, \mathrm{Wu} X, \mathrm{Wu}$ J, et al. Delivering PD-1 inhibitory signal concomitant with blocking ICOS costimulation supresses lupus-like syndrome in autoimmune BXSB mice. Clin Immunol. 2006; 118: 258-267.

62. Hirata S, Senju S, Matsuyoshi H, et al. Prevention of experiment autoimmune encephalomyelitis by transfer of embryonic stem cell-derived dendritic cells expressing myelin oligodendrocyte glycoprotein peptide along with TRAIL or programmed death-1 ligand. J Immunol. 2005; 174: 1888-1897.

63. Schreiner B, Mitsdoerffer M, Kieseier BC, et al. Interferon-beta enhances monocyte and dendritic cell expression of B7-H1 (PD-L1), a strong inhibitor of autologous T-cell activation: relevance for the immune modulatory effect in multiple sclerosis. J Neuroimmunol. 2004; 155: 172-182.

64. Zhou Q, Xiao H, Liu Y, et al. Blockade of programmed death -1 pathway rescues the effector function of tumor-infiltrating $\mathrm{T}$ cells and enhances the antitumor efficacy of lentivector immunization. J Immuno. 2010; 185: 5082-5092.

65. Sierro SR, Donda A, Perret R, et al. Combination of lentivector immunization and low-dose chemotherapy of PD-1/PD-L1 blocking primes self-reactive $\mathrm{T}$ cells and induces anti-tumor immunity. Eur J Immunol. 2011; 41: 2217-2228

66. Wong RM, Scotland RR, Lau RL, et al. Programmed death-1 blockade enhances expansion and functional capacity of human melanoma antigen-specific CTLs. Int Immunol. 2007; 19: 1223-1234.

67. Borkner L, Kaiser A, van de Kasteele W, et al. RNA interference targeting programmed death receptor-1 improves immune function of tumor-specific T cells. Cancer Immunol Immunother. 2010; 59: 1173-1183.

68. Iliopoulos D, Kavousanaki M, Ioannou M, et al. The negative costimulatory molecule PD-1 modulates the balance between immunity and tolerance via miR-21. Eur J Immunol. 2011; 41: 1754-1763.

69. Brahmer JR, Tykodi SS, Chow LQM, et al. Safety and activity of anti-PD-1 antibody in patients with advanced cancer. N Engl J Med. 2012; 366: 2455-2465.

70. Topalian SL, Hodi FS, Brahmer JR, et al. Safety, activity, and immune correlates of anti-PD-L1 antibody in cancer. N Engl J Med. 2012; 366: 2443-2454.

71. Pardoll DM. The blockade of immune immunotherapy. Nat Rev Cancer. 2012; 12: 252-264.

72. Day CL, Kaufmann DE, Kiepiela P, et al. PD-1 expression on HIV-specific $\mathrm{T}$ cells is associated with T-cell exhaustion and disease progression. Nature. 2006; 443: 350-354.

73. Ma CJ, Ni L, Zhang Y, et al. PD-1 negatively regulates interleukin-12 expression by limiting STAT-1 phosphorylation in monocytes/macrophages during chronic hepatitis $\mathrm{C}$ virus infection. Immunology. 2010; 132: 421-431.

74. Blackburn SD, Shin H, Haining $\mathrm{WN}$, et al. Coregulation of $\mathrm{CD}^{+} \mathrm{t}$ Cell exhaustion during chronic viral infection by multiple inhibitory receptors. Nat Immunol. 2009; 10: 29-37.

75. Das S, Suarez G, Beswick EJ, et al. Expression of B7-H1 on gastric epithelial cells: its potential role in regulating $\mathrm{T}$ cells during Helicobacter pylori infection. J Immunol. 2006; 176: 3000-3009.

76. Smith P, Walsh CM, Mangan NE, et al. Schistosoma mansoni worms induce anergy of $\mathrm{T}$ cells via selective up-regulation of programmed death ligand 1 on macrophages. J Immunol. 2004; 173: 1240-1248.

77. Monaghan SF, Thakkar RK, Chung CS, et al. Lack of programmed cell death receptor (PD)-1 leads to improved survival in a murine model of indirect acute lung injury. J Am Coll Surg. 2010; 211: s52.

78. Peña-Cruz V, McDonough SM, Diaz-Griffero F, et al. PD-1 on immature and PD-1 ligands on migratory human langerhans cells regulate antigenpresenting cell activity. J Invest Dermatol. 2010; 130: 2222-2230.

79. Özkaynak E, Wang L, Goodear A, et al. Programmed death-1 targeting can promote allograft survival. J Immunol. 2002; 169: 6546-6553.

80. Blazar BR, Carreno BM, Panoskaltsis-Mortari A, et al. Blockade of programmed death-1 engagement accelerates graft-versus-host disease lethality by an IFN-gamma-dependent mechanism. J Immunol. 2003; 171: 1272-1277.
81. Gao W, Demirci G, Strom TB, et al. Stimulating PD-1-negtive signals concurrent with blocking CD154 co-stimulation induces long-term islet allograft survival. Transplantation. 2003; 76: 994-999.

82. Bernard D, Hansen JD, Pasquier LD, et al. Costimulatory reseptors in jawed vertebrates: conserved CD28, odd CTLA-4 and multiple BTLAs. Dew Comp Immunol. 2007; 31: 255-271.

83. Chemnitz JM, Lanfranco AR, Braunstein I, et al. B and T lymphocyte attenuator-mediated signal transduction provides a potent inhibitory signal to primary human CD4 $\mathrm{T}$ cells that can be initiated by multiple phosphotyrosine motifs. J Immunol. 2006; 176: 6603-6614.

84. Gonzalez LC, Loyet KM, Calemine-Fenaux J, et al. A co-receptor interaction between the CD28 and TNF receptor family members B and T lymphocyte attenuator and herpesvirus entry mediator. PNAS USA. 2005; 102: 1116-1121.

85. Watanabe N, Gavrieli M, Sedy JR, et al. BTLA is a lymphocyte inhibitory receptor with similarities to CTLA-4 and PD-1. Nat Immunol. 2003; 4: 670-679.

86. Compaan DM, Gonzalez LC, Tom I, et al. Attenuationg lymphocyte activity-the crystal structure of the BTLA-HVEM complex. J Biol Chem. 2005; 280: 39552-39561.

87. Krieg C, Boyman O, Fu YX, et al. B and T lymphocyte attenuator regulates $\mathrm{CD}^{+} \mathrm{T}$ cell intrinsic homeostasis and memory cell generation. Nat Immunol. 2007; 8: 162-171.

88. Sedy JR, Gavrieli M, Potter KG, et al. B and T lymphocyte attenuator regulates $\mathrm{T}$ cell activation through interaction with herpesvirous entry mediator. Nat Immunol. 2005; 6: 90-98.

89. Hurchla MA, Sedy JR, Gavrielli M, et al. B and T lymphocyte attenuator (BTLA) exhibits structure and expression polymorphisms and is highly induced in anergic CD4 ${ }^{+}$T cells. J Immunol. 2005; 174: 3377-3385.

90. Hurchla MA, Sedy JR, Murphy KM. Unexpected role of B and T lymphocyte attenuator in sustaining cell survival during chronic allostimulation. J Immunol. 2007; 178: 6073-6082.

91. Lin SC, Kuo CC, Chan CH. Association of a BTLA gene polymorphism with the risk of rheumatoid arthritis. J Biomed Sci. 2006; 13: 853-860.

92. Fu Z, Li D, Jiang W, et al. Association of a BTLA gene polymorphism with the risk of malignant breast cancer in Chinese women of Heilongjiang Province. Breast Cancer Res Treat. 2010; 120: 195-202.

93. Wang Y, Subudhi SK, Anders RA, et al. The role of herpesvirus entry mediator as a negative regulator of $\mathrm{T}$ cell-mediated responses. J Clin Invest. 2005; 115: 711-717.

94. Lin SC, Kuo CC, Chan CH. Association of a BTLA gene polymorphism with the risk of rheumatoid arthritis. J Biomed Sci. 2006; 13: 853-860.

95. Oya Y, Watanabe N, Owada T, et al. Development of autoimmune hepatitis-like disease and autoantibody production to nuclear antigens in mice lacking B and T lymphocyte attenuator (BTLA). Arthritis Rheum. 2008; 58: 2498-2510.

96. Oya Y, Watanabe N, Kobayashi Y, et al. Lack of B and T lymphocyte attenuator exacerbates autoimmune disorders and induces Fas-independent liver injury in MRL-Ipr/lpr mice. Int Immunol. 2011; 23: 335-344.

97. Pierer M, Schulz A, Rossol M, et al. Herpesvirus Entry Mediator-Ig Treatment during Immunization Aggravates Rheumatoid Arthritis in the Collagen-Induced Arthritis Model. J Immunol. 2009; 182: 3139-3145.

98. Derré L, Rivals JP, Jandus C, et al. BTLA mediated inhibition of human tumor-specific CD8 ${ }^{+} \mathrm{T}$ cells that can be partially reversed by vaccination. J Clin Invest. 2010; 120: 157-167.

99. Lepenies B, Pfeffer K, Hurchla MA, et al. Ligation of B and T Lymphocyte Attenuator Prevents the Genesis of Experimental Cerebral Malaria. J Immunol. 2007; 179: 4093-4100.

100. Tao R, Wang L, Han R, et al. Differential Effects of B and T Lymphocyte Attenuator and Programmed Death-1 on Acceptance of Partially versus Fully MHC-Mismatched Cardiac Allografts. J Immunol. 2005; 175: 5774-5782.

101. Granger SW, Rickert S. LIGHT-HVEM signaling and the regulation of T cell-mediated immunity. Cytokine Growth Factor Rev. 2003; 14: 289-296.

102. Truong W, Plester JC, Hancock WW, et al. Combined Coinhibitory and Costimulatory Modulation with Anti-BTLA and CTLA4Ig Facilitated Tolerance in Murine Islet Allografts. Am J Transplant. 2007; 7: 2663-2674.

\section{Author biography}

Dr. Yan-Ling $\mathbf{W u}$ is a professor in Molecular Immunology and now heads the Cellular and Molecular Immunology Research Group. She received Master and Doctoral degrees in Applied Life Science 
in 2003 and in Medicine Science in 2006, respectively, from Tohoku University, Japan. After that, she entered to Professor Minato's group of School of Medicine, Kyoto University, Japan, as a senior researcher working in the field of molecular immunology. Her current researches focus on understanding the molecular mechanisms of gene regulation related to diseases by immune inhibitory receptors. Dr. Wu have given oral presentations in international conferences and published related papers.

Dr. Wen Zhang is a professor with 25 years of research and teaching experience in Bioorganic Chemistry and Chemical Biology. Dr. Zhang obtained a Doctorate degree in Bioorganic Chemistry from East China University of Science and Technology, China. Then, he entered to Professor Ohrui's Lab of Tohoku University, Japan, working in the field of molecular recognition as a JSPS postdoctoral fellow. After that, he joined Professor Sugiyama's Chemical Biology group of Kyoto University as a COE and JST research fellow working on biology and chemistry of polyamide-nucleic acids interaction. Now, Dr. Zhang has a special interest in elucidating the gene regulation mechanisms with organic small molecules and the development of gene-targeted drug. His group formed in 2008 and established an extremely fruitful collaboration with Prof. Sugiyama's Group in order to better pursue aspects of gene-targeted drug research. To date, Dr. Zhang has published better papers in excellent Journals including JACS, ChemBioChem etc.

Jing Liang is a postgraduate majoring in pharmacognosy. She obtained the Bachelor's Degree in Biotechnology in 2010 from Guilin Medical University, China. Then, she entered Prof. Zhang's group of College of Pharmaceutical Sciences, Zhejiang University of Technology, China in 2010. She is working with small molecules regulating disease-related gene to explore gene-targeted drugs under the direction of Profs W. Zhang and Y.-L. Wu. 